\title{
ROLE OF FREE / TOTAL PSA RATIO TO DIFFERENTIATE BPH AND PROSTATE CANCER
}

\section{GEETA RATHNAKUMR ${ }^{\mathrm{a} 1}$, NITIN INAMDAR ${ }^{\mathrm{b}}$ AND KINJALKA GHOSH}

${ }^{a b c}$ Department of Biochemistry, Tata Memorial Hospital, E.S. Patanwala Marg, Parel, Mumbai, Maharashtra, India

\begin{abstract}
This study was undertaken to determine Free to Total PSA ratio to differentiate between Benign Prostate Hyperplasia (BPH) and Prostate Cancer (PCa) and to improve the accuracy of the PSA test and the specificity of PCa detection particularly when PSA levels fall between 4-10ng/ml levels. The study group consisted of a total of 124 subjects. Free PSA and Total PSA were determined using assay, which is based on Chemiluminescent Microparticle Immunoassay (CMIA) technology. The Statistical test used were Students' t test for difference of Mean and Pearson Correlation test, using SPSS and MS Excel Statistical packages. Free/Total PSA ratios in the prostate cancer group were significantly lower than those in the BPH group. A cut-off of F/T PSA ratio\% at $18 \%$ improves diagnostic sensitivity and specificity for prostate cancer.
\end{abstract}

KEYWORDS: Benign Prostate Hyperplasia (BPH), Prostate cancer (Pca), Free PSA, Total PSA, Chemiluminescent Microparticle Immunoassay (CMIA)

$\mathrm{PCa}$ is the third most common cause of death from cancer in men of all ages. It is the most prevalent cancer found in men above the age of fifty year and is frequently diagnosed in men (Malati and Rajanikumar, 2006) between 45 and 90 years of age with a median age of 72 years. The age of Indian patients of $\mathrm{PCa}$ varies from 35-86, which is much lower when compared with average age of patients in western countries. The actual incidence of prostate cancer in India and in nonresident Indians is lower than that in the Western populations (Krishnamoorthy and Padmanabha, 2016). According to a recent study, PCa ranks fifth in incidence and fourth in cancer mortality for men in Mumbai and the incidence is increasing by $1 \%$ every year (Yeole, 2008). Fifty to sixty percent of men over the age of 60 in India have Benign Prostate hyperplasia (BPH). 30-40\% show related symptoms and $10 \%$ out of this group may have PCa. BPH affects 40 percent of men in their 50s and 90 percent of men in their $80 \mathrm{~s}$. BPH is a noncancerous enlargement of the prostate gland that may restrict the flow of urine from the bladder. The possibility of having PCa varies with age, being $13.7 \%$ for the age group $60-79$ years, $2.2 \%$ for those aged $40-59$, and $0.005 \%$ in those younger than 39 years. Men who have a brother or father with $\mathrm{PCa}$ have twice the usual risk of developing $\mathrm{PCa}$ (Steinberg et al., 1990)' Gene alterations on chromosome 1, 17, and the $\mathrm{X}$ chromosome have been found in some patients with a family history of PCa. African American men have a higher prevalence and more aggressive prostate cancer than white men, who, in turn, have a higher prevalence than men of Asian origin (Hoffman et al., 2001). Men with higher serum levels of the short-chain omega-6 fatty acid linoleicacid have higher rates of PCa. However, the same series of studies showed that men with elevated levels of long-chain omega-3 fatty acids (EPA and DHA), found in fatty fishes like salmon had lowered incidence (Gann and Giovannucci, 2005). Other dietary factors that may increase PCa risk include low intake of vitamin $\mathrm{E}$ and the mineral selenium. Decreased levels of vitamin A may be a risk factor because this can promote cell differentiation and stimulate the immune system. Vitamin D deficiency was suggested as a risk factor, and studies show an inverse relationship between ultraviolet exposure and mortality rates for $\mathrm{PCa}$ (Peters et al., 2007).

$\mathrm{PCa}$ can also be attributed to hormonal reasons. For instance, androgen ablation causes a regression of PCa. Obesity and elevated blood levels of testosterone might increase the risk for PCa. Daily use of anti-inflammatory medicines such as aspirin, ibuprofen, or naproxen may decrease PCa risk. Use of the cholesterol-lowering drugs known as the statins may also decrease PCa risk (Hsing and Comstock, 2009) (Jacobs et al., 2005) (Shannon et al., 2005) (Platz et al., 2005).

More frequent ejaculation also may decrease a man's risk of PCa (Giles et al., 2003). The researchers hypothesize that this could be because regular ejaculation reduces the buildup of carcinogenic deposits such as 3methylcholanthrene, produced from the breakdown of cholesterol, which could damage the cells lining the prostate. 


\section{Symptoms for PCa}

Early diagnosis of $\mathrm{PCa}$ is hindered by lack of symptoms in men with localized tumors. Some men, however, will experience symptoms such as frequent, hesitant, or burning urination, difficulty in having an erection, or pain or stiffness in the back, hips or upper thighs. Some advanced PCa can slow or weaken the urinary stream or increase the need to urinate more often. But noncancerous diseases of the prostate, such as BPH are a more common cause of these symptoms. If the $\mathrm{PCa}$ is advanced, one might develop blood in the urine (hematuria) or might find it difficult to get an erection (impotence). Advanced PCa commonly spreads to the bones, which can cause pain in the hips, spine, ribs, or other areas (Springhouse, 2005). Cancer that has spread to the spine can also cause it to press on the spinal nerves, which can result in weakness or numbness in the legs or feet, or even loss of bladder or bowel control.

\section{Diagnosis of Prostate Cancer} initially.

The following screening tests may be used

1. Digital rectal examination (DRE): Data from community-based studies suggest that the positive predictive value of digital rectal examination for prostate cancer is $15 \%$ to $30 \%$ and varies relatively little with age (Chodak et al., 1989).

2. Prostate Specific Antigen (PSA) Test: If higher than normal levels of PSA are detected or if levels rise over time, it could indicate prostate inflammation, prostate enlargement or prostate cancer (Brawer et al., 1992).

3. Ultrasound (Transrectal Ultrasonography): A transrectal ultrasonography (TRUS) provides a visual image of the prostate and is used if the DRE indicates the presence of cancer, the procedure may help to confirm an uncertain preliminary diagnosis and is useful as a guide for needle biopsies (Gupta et al., 2005).

4. Biopsy: This is more of a confirmation diagnosis.

These tests are also used to determine which tumors are localized within the prostate and are, thus, potentially treatable. Observational studies suggest that DRE and PSA, combined with TRUS and biopsy, can identify localized prostate cancer in $3-5 \%$ of men, although the tests do result in a number of false positives and negatives.

\section{Treatment for Prostate Cancer}

Three major treatment options are available for localized prostate cancer: radical prostatectomy, radical radiotherapy and conservative management (involving monitoring and treatment of symptoms) (Milin, 1947).

\section{PSA}

PSA has been characterized as the most useful serum marker for the detection and management of $\mathrm{PCa}$, however, Screening with PSA is problematic because it is really an organ specific marker for the prostate rather than a specific marker for cancer. There is considerable overlap in PSA concentration in men with benign prostate disease. In this regard, the recognition of the molecular forms of PSA, Free PSA has shown the most promise. Although PSA is considered to be the most effective tumor marker in human oncology, its role in screening strategies for PCa has not been completely established. It is a glycoprotein produced exclusively by the epithelial cells lining the prostatic ducts and acini. Normally, it is secreted into the prostatic ducts and is present only in prostate tissue, prostatic fluid, and seminal plasma. PSA is produced by normal, hyperplastic, and cancerous prostatic tissue (Emil and Jack). A major function of PSA is proteolytic cleavage of gel forming proteins in the seminal fluid, resulting in the liquification of the seminal gel and increased sperm mobility.

\section{PSA and Protease Inhibitors}

PSA circulates in serum in complex forms (bound to protease inhibitors) or in non-complex (free or unbound) forms. Approximately $60 \%$ to $70 \%$ of PSA occurs in an active free form (Zhang et al., 1998) with less than $5 \%$ in complex with protease inhibitors. The major protease inhibitor, to which active single-chain PSA is covalently linked in a 1:1 molar ratio, is PCI where by the PSA-PCI complex could only be detected in seminal plasma and not in serum.

\section{PSA and Protease Inhibitors in Blood}

PSA occurs in three major forms in the blood. The major immunodetectable form is PSA complexed with the serine protease inhibitor and alpha-1-antichymotripsin (PSA-ACT). In contrast to seminal fluid, $55 \%$ to $95 \%$ of immunodetectable PSA forms covalent 1:1 molar 
complexes with ACT. Uncomplexed, or FREE PSA, is the other immunodetectable form of PSA in serum between $5 \%$ to $45 \%$ of the immunodetectable PSA is found in a free noncomplexed form, which is most likely catalytically inactive as it remains virtually nonreactive with the large excess of protease inhibitors (ACT or AMG) in blood. A third form of PSA, a complex with alpha-2-macroglobulin (AMG) is not detectable with current immunoassays for PSA due to the engulfment and subsequent masking of PSA epitopes by the alpha-2-macroglobulin molecule (Stephan et al., 1981).The serum concentration of the PSAAMG complex is difficult to assess, likely due to the conformation of the AMG molecule that engulfs the PSA molecule, hence blocking antibody access to the PSA epitopes.

PSA level in the blood is found if the barrier between the epithelium and the blood stream is damaged. Typical sources for damage are cancer, bacterial infection, and prostate infection or destruction of part of the prostate by damage to its blood supply. PSA is also present in urine and serum. Low levels of PSA are found in the blood as a result of leakage of PSA from the prostate gland. Increasing levels of PSA are associated with prostatic pathology; including prostatitis, BPH, and PCa (Lija et al., 1999). Numerous studies have shown that for groups of men with PCa, serum PSA correlates directly with pathologic stage. However, in most cases, PSA level alone does not provide accurate information because of overlap in PSA level between stages. False positive elevations in the PSA increases in the PSA that are caused by conditions other than PCa for example BPH and infection or prostates can elevate the PSA (Ornstein et al., 1998).

\section{Role of PSA in Pca}

In general, normal PSA levels are below $4 \mathrm{ng} / \mathrm{ml}$. A reading between 4 and $10 \mathrm{ng} / \mathrm{ml}$ indicates about $20 \%$ chance that cancer is present and a measurement above $10 \mathrm{ng} / \mathrm{ml}$ is considered a strong indication of PCa (Catalona and Scoott, 1986). But because various factors can cause PSA levels to fluctuate, one abnormal PSA test does not necessarily indicate that cancer is present. Once cancer is diagnosed, PSA level is also measured to determine its extent. If PSA level is less than $20 \mathrm{ng} / \mathrm{ml}$ then it is likely the cancer has not spread. Metastasis is strongly indicated as the level rises over $40 \mathrm{ng} / \mathrm{ml}$. PSA level is also used for monitoring the course of the disease after initial treatment of prostatic cancer. Rising level indicates recurrence of tumour, although the exact location cannot be determined just by using PSA. In contrast to this, if the PSA falls significantly after treatment and remains persistently low, then it will indicate the disease is under control (Lodding et al., 1998).

The present study is undertaken with a view to determine Free to Total PSA ratio to differentiate between $\mathrm{BPH}$ and PCa and to improve the accuracy of the PSA test and the specificity of PCa detection particularly when PSA levels fall between $4-10 \mathrm{ng} / \mathrm{ml}$ levels.

\section{MATERIALS AND METHODS}

- Target population was male referred to genitourinary unit of our Institute between the age group 50-90 yrs. In the duration of course the medical records of histological proven patients were studied. The blood sample were collected from these patients. Normal control blood were taken from 30 healthy ambulant donors. Serum were extracted by centrifugation of samples at 4000rpm for $10 \mathrm{~min}$, and were stored at -20 ${ }^{\circ} \mathrm{C}$ until use.

\section{Methods}

- Serum levels of Free PSA and Total PSA were determined using assay, which is based on Chemiluminescent Microparticle Immunoassay (CMIA, Marketed) technology. The Architect i system and reagent kit from Abbott were used.

- The Statistical test used were Students' $t$ test for difference of Mean and Pearson Correlation test, using SPSS and MS Excel Statistical packages.

\section{RESULTS AND DISCUSSION}

The study group consisted of 104 male patients comprising of $54(57 \%)$ PCa patients and $40(43 \%)$ patients with BPH. The mean age for these groups was $65.90 \pm 8.11$ and $67.32 \pm 7.54$ respectively. 30 age matched healthy male formed the control group with the mean age of $60.90 \pm$ 7.17. For the three groups viz. Normal cases, BPH patients and PCa patients, the data was analyzed for FPSA, TPSA and F/T PSA\%. Following is the tabulation for the data. 
RATHNAKUMR ET AL.: ROLE OF FREE / TOTAL PSA RATIO TO DIFFERENTIATE BPH AND PROSTATE CANCER

Table 1: Normal cases, BPH patients and PCa patients

\begin{tabular}{|c|c|c|c|}
\hline \multicolumn{5}{|c|}{ Normal Healthy controls } \\
\hline & FPSA ng/ml & TPSA ng/ml & (FPSA)/(TPSA) \% \\
\hline Range & 0.040 to 1.162 & 0.147 to 4.00 & $25.98-58.04$ \\
\hline Mean & 0.38 & 1.12 & 37.17 \\
\hline Standard Deviation & 0.26 & 0.68 & 10.66 \\
\hline \multicolumn{5}{|c|}{ Benign Prostate Hyperplasia } \\
\hline Range & FPSA ng/ml & TPSA ng/ml & (FPSA)/(TPSA) \% \\
\hline Mean & 0.386 to 3.396 & 2.075 to 16.816 & $10.47-54.6$ \\
\hline Standard Deviation & 1.75 & 7.54 & 24.68 \\
\hline \multicolumn{5}{|c|}{ Prostate Cancer } & 4.64 & 10.56 \\
\hline Range & FPSA ng/ml & TPSA ng/ml & (FPSA)/(TPSA) \% \\
\hline Mean & 0.052 to 2.937 & 2.078 to 30.0 & $4.06-20.85$ \\
\hline Standard Deviation & 1.02 & 9.29 & 10.91 \\
\hline
\end{tabular}

We note that the mean TPSA levels are the least for the healthy patients and it increases in diseased condition being higher in prostate cancer patient. We also note that the F/T PSA \% ratio were significantly different amongst the three groups that we have at a significance level $(\mathrm{p}<0.001)$.Percentage $\mathrm{F} / \mathrm{T}$ PSA levels ranged from 10.47 to $54.6 \%$ (mean \pm SE, $24.68 \pm 10.56$ ) and from 4.06 to $20.85 \%$ (mean $\pm \mathrm{SE}, 10.9 \pm 6.92$ ) in patients with $\mathrm{BPH}$ and $\mathrm{PCa}$, respectively. The mean F/T PSA ratio was found to be significantly lower in the group of patients with prostate cancer. This finding is consistent with the findings of Filella et al., (1997).

Our findings are consistent with the study done by $\mathrm{V}$ Thakur et al. wherein they discussed the utility of F/T PSA ratio in diagnosis of prostate carcinoma. They found that mean total PSA was the least in normal healthy subjects and was the highest in the cancer patients. Further they also found that the F/TPSA ratio was significantly different between the three groups. Recently numerous studies have demonstrated a lower ratio of FPSA/TPSA in Ca P patients compared with patients with BPH (Brawer et al., 1992) (Gupta et al., 2005) (Milin, 1947)

If we look at the frequency distribution of no. of cases of Normal, BPH and Cancer across various values of Total PSA, as shown in the graph below:

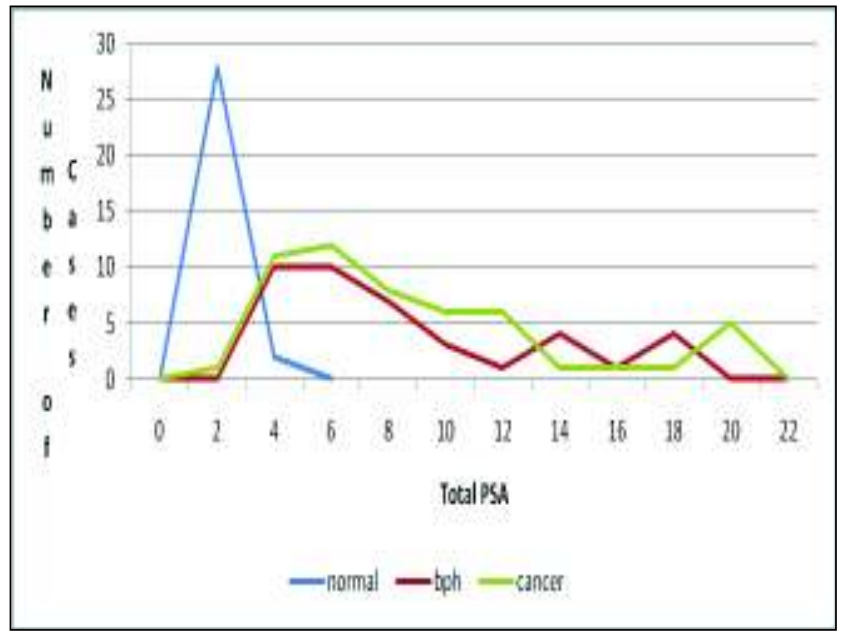

Figure 1: BPH and Cancer across various values of Total PSA

We find that there is an area of overlap between BPH and Cancer between the Total PSA values of 4 to 10 ; the grey zone. Theoretically, the occurrence of the various PSA forms in serumallows for a better discrimination between prostate cancer and BPH (Malati and Rajanikumar, 2006).

If we do the two tailed t-test for difference in mean between Cancer, BPH and control (normal) we find that there is significant difference between BPH $(24.68 \pm 10.56)$ and control (normal) $(37.17 \pm 10.66, \mathrm{p}=0.00)$ and between Cancer ( $10.91 \pm 6.92)$ and control (normal) (37.17 \pm 10.66 , 
$\mathrm{p}=0.00$ ) (significance level $\alpha=0.05$, using student's t test for difference of means)

Now if we replicate a similar test on the Cancer and $\mathrm{BPH}$ groups to find if there is a significant difference between the mean of two groups we find that they are significantly different with the value of $p=0.000$ at a significance level of $\alpha=0.05$. This establishes that the ratio (FPSA/TPSA) \% can be used to identify cases of Cancer from BPH in the grey zone (TPSA from 4 to $10 \mathrm{ng} / \mathrm{ml}$ )

The study was performed on 94 consecutive male patients consisting of 40 patients with $\mathrm{BPH}$ and $54 \mathrm{PCa}$ patients. The sensitivity and specificity was calculated at different \%FPSA/TPSA ratio cutoff. From cutoff value 16 to 22 , sensitivity increased from $86 \%$ to $96 \%$, but specificity decreased from $98 \%$ to $80 \%$. Increasing the cutoff value thereafter showed no change in sensitivity, but specificity further reduced to $65 \%$. Hence in this patient's series we find that at $18 \%(\% \mathrm{FPSA} / \mathrm{TPSA})$ we get the maximum sensitivity with a stable level of specificity.

Also if we look at plot of positive predictive and negative predictive value vs \%FPSA/TPSA we find that at $18 \%$ point we have a very high level of "positive predictive value" and a relatively high and stable level of "negative predictive value". The trend is evident even in the plot of "False negative" and "False positive" vs \%FPSA/TPSA plot. Here again we see that at $18 \%$ point, the "False negative" is the lowest with a relatively very low and stable level of "False positive" cases

Hence we can see that $18 \%$ ratio FTPSA/TPSA can be taken as a cutoff value to differentiate between $\mathrm{BPH}$ and PCa cases; any value of the \% ratio FPSA/TPSA lower than $18 \%$ is indicative of a PCa case and any value higher than $18 \%$ is indicative of $\mathrm{BPH}$.

The cutoff found here is similar to the findings of a study conducted in Shaikh Zayed hospital \& Mayo hospital by Saima Naz, Sarah Ahmad et al., wherein they found that at the $18 \%$ cutoff point, the sensitivity was $86 \%$ and specificity was $94 \%$. Using a cutoff of $18 \%$, the free-PSA test significantly improved the differential diagnosis of $\mathrm{BPH}$ and $\mathrm{Ca} \mathrm{P}$ in the 'gray zone' as compared to the use of total PSA alone in the study group.

In a case controlled study of 200 patients conducted by Iqbal et al., at Jinnah hospital, Lahore, found that at $18 \%$ cutoff, the test gives a specificity and sensitivity of $91.9 \%$ and $92.1 \%$ respectively with $87.2 \%$ PPV. Further concurrence on $18 \%$ cutoff for the test was found with a study done by Gaspar and Arribas in 2000, Madrid hospital.

Numerous retrospective studies demonstrated that $\sim 19-64 \%$ of unnecessary, negative biopsies could be eliminated (Jacobs et al., 2005) (Brawer et al., 1992) (Stephan et al., 1981) Reasons for these substantial differences could be the use of a wide range of $\mathrm{PPSA} \%$ cutoffs (14-28\%) with different assay.

Currently there are many different recommendations and different cutoffs for the use of fPSA \% depending on the various assays used and on the targeted sensitivity or specificity, the reported rates of sensitivity, specificity, and predictive values are widely scattered (Chodak et al., 1989) (Brawer et al., 1992). The analytical variability of assay methods could be the cause of discrepancies among the results of different studies (Milin, 1947). But, let us evaluate the strength of the test based on the $\%$ FPSA/TPSA with cut off $18 \%$ on the patient data that we have. (Table 2)

Table 2: Percentage of FPSA/TPSA

\begin{tabular}{|l|c|c|c|}
\hline TPSA>=4 & BPH & Cancer & Total \\
\hline Actual & 30 & 41 & 71 \\
\hline TPSA & 0 & 71 & 71 \\
\hline \%FPSA/TPSA (18\%) & 28 & 43 & 71 \\
\hline & \multicolumn{2}{|c|}{$\begin{array}{c}\text { False } \\
\text { negative }\end{array}$} & $\begin{array}{c}\text { False } \\
\text { positive }\end{array}$ \\
\hline TPSA (>=4) & \multicolumn{2}{|c|}{0} & 30 \\
\hline \%FPSA/TPSA (18\%) & 0 & 2 \\
\hline
\end{tabular}

If we first apply the TPSA test, then all the cases where TPSA $>=4,71$ out of 124 , will be indicative for biopsy. From the actual data, we know that only 41 out of these 71 are actual positive cancer cases. This means that 30 cases of those sent for biopsy are only BPH and negative for $\mathrm{PCa}$.

Now, if we apply our \%FPSA/TPSA ratio test with a cutoff of $18 \%$, then only those cases (amongst those with TPSA $>=4$ ) where the ratio $<=18 \%$ will be sent for biopsy i.e. 43. Comparing it with actual data we find that of the 43 cases sent for biopsy only 2 cases are those which are only $\mathrm{BPH}$ and negative for prostate cancer. 
Comparing 30 false positive cases versus only 2 false positive cases, we can easily conclude that employing the ratio test along with the TPSA test increases the effectiveness of differentiating cases of BPH from positive cancer cases. We further try the ratio test with $18 \%$ cutoff on the "Grey zone" or overlap between BPH and Cancer cases which is between $4<=$ TPSA $<10$ and we have the following results (Table 3)

Now, if we apply our \%FPSA/TPSA ratio test with a cutoff of $18 \%$ to the grey zone (TPSA $4-10 \mathrm{ng} / \mathrm{ml}$ )

Table 3: TPSA Test

\begin{tabular}{|l|c|c|c|}
\hline $\mathbf{4}<=$ TPSA $<\mathbf{1 0}$ & BPH & Cancer & Total \\
\hline Actual & 20 & 26 & 46 \\
\hline TPSA & & 46 & 46 \\
\hline \%FPSA/TPSA (18\%) & 19 & 27 & 46 \\
\hline & $\begin{array}{c}\text { False } \\
\text { negative }\end{array}$ & \multicolumn{2}{|c|}{$\begin{array}{c}\text { False } \\
\text { positive }\end{array}$} \\
\hline TPSA (>=4) & 0 & \multicolumn{2}{|c|}{20} \\
\hline \%FPSA/TPSA (18\%) & 0 & \multicolumn{2}{|c|}{1} \\
\hline
\end{tabular}

In the above tables we can easily see only a TPSA test is unable to differentiate between cases of BPH and Cancer. But if we apply the ratio test with cutoff of $18 \%$ we find that, it detects $19 \mathrm{BPH}$ cases compared to 20 as per actual data and 27 cancer cases compared to 26 as per actual data. So we can conclude that the \%ratio FPSA/TPSA test, with a cutoff of $18 \%$, is highly effective at differentiating BPH cases from cancer cases in the "grey zone".

We found that the percent free PSA was not associated with patientage, in agreement with Oesterling et al., Filella et al., Morgan et al., and Jung et al. A recent, prospective study by Catalona et al., showed a statistically significant and clinically relevant direct association between percent free PSA and age. (Table 4)

Now, if we apply our \%FPSA/TPSA ratio test with a cutoff of $18 \%$ to the cases where TPSA $<=4 \mathrm{ng} / \mathrm{ml}$
Table 4: Percentage FPSA/TPSA ratio test

\begin{tabular}{|l|c|c|c|}
\hline TPSA $<4$ & $\begin{array}{c}\text { Non } \\
\text { Cancer }\end{array}$ & Cancer & Total \\
\hline Actual & 40 & 12 & 52 \\
\hline TPSA & 52 & & 52 \\
\hline \%FPSA/TPSA (18\%) & 40 & 12 & 52 \\
\hline & $\begin{array}{c}\text { False } \\
\text { negative }\end{array}$ & $\begin{array}{c}\text { False } \\
\text { positive }\end{array}$ \\
\hline TPSA (>=4) & \multicolumn{2}{|c|}{12} & 0 \\
\hline \%FPSA/TPSA (18\%) & 0 & 0 \\
\hline
\end{tabular}

For the cases where TPSA $<4$, if we use only TPSA as a test, all the cases would be diagnosed as negative for cancer and none would be sent for biopsy as a result. From the actual data we find that this would be a wrong diagnosis for 12 of the cases, which should have been sent for Biopsy. However, if we use \%FPSA/TPSA test with a cut off of $18 \%$, as found earlier, we would be correctly identifying all the 12 cases as positive for cancer and would be rightly sending them for biopsy.

Hence we see that for cases where TPSA $<4$, the test $\% \mathrm{FPSA} / \mathrm{TPSA}$ enhances the sensitivity of detecting the cancer patients. Needless to say it will help in early detection for cancer.

\section{CONCLUSION}

In conclusion, F/T PSA ratios in the $\mathrm{PCa}$ group were significantly lower than those in the BPH. A cut-off of F/T PSA ratio $\%$ at $18 \%$ improves diagnostic sensitivity and specificity for PCa.

The results from this study show that the use of the percentage FPSA value ratio improves PSA-based differential diagnosis of $\mathrm{PCa}$ in patients and help differentiate $\mathrm{PCa}$ from $\mathrm{BPH}$ and reduce unnecessary prostate biopsies. Selective measurement of percent free PSA in cases of uncertain diagnosis can improve the specificity of PCa detection especially when the TPSA falls between 4-10ng/ml thus making F/T PSA ratio an excellent adjuvant to TPSA for diagnosis of $\mathrm{PCa}$, increasing the specificity and predictive value for positive test which in turn will assist the physician in determining the probability of $\mathrm{PCa}$, managing the patient and taking informed care 


\section{RATHNAKUMR ET AL.: ROLE OF FREE / TOTAL PSA RATIO TO DIFFERENTIATE BPH AND PROSTATE CANCER}

decision. In the near future, the FPSA\% value will be used more frequently with additional clinical and laboratory values to calculate the risk of having cancer.

In conclusion, we predict that better insight in regard to PSA molecular forms will lead not only to better diagnostic markers, but also to an understanding of $\mathrm{PCa}$ development and possible new therapeutic options.

FPSA measurements and the use of its ratio to TPSA are well established in numerous, large-scale, multiinstitutional clinical trials. A forthcoming standardization of the FPSA and TPSA measurements will provide a better comparability of different PSA assays. In the near future, the FPSA \% value will be used more frequently with additional clinical and laboratory values to calculate the risk of having cancer.

\section{REFERENCES}

Brawer M.K., Chetner M.P., Beatie J., Buchner D.M., Vessella R.L. and Lange P.H., 1992. Screening for Prostatic carcinoma with prostate specific antigen: J. Urol., 147: 841-5.

Catalona W.W.J. and Scoott W.W., 1986. Carcinoma of the prostate. In Walsh P.C., Gittes R.F., Perlmuuter Ad., Stamey T.A.: Campbell's Urology, $5^{\text {th }}$ edition. 1463: Philadelphia.

Chodak G.W., Keller P. and Schoenberg H.W., 1989. Assessment of screening for prostate cancer using the digital rectal examination: J. Urol., 141: 11368.

Emil A. Tanagho and Jack W. McAninch: Smith's general urology: Page 369.

Gann Ph. and Giovannucci, 2005. "Proatate cancer and Nutrition" (PDF). http://www.prostatecancer foundation.org/atr/cf\%B705B3273-F2EF-4EF6A653-E15C5D8BB6B1\%7D/Nutrition_Guide.pdf. 20:2006.

Giles G.G., Severi G., English D.R., McCredie M.R., Borland R., Boyle P. and Hopper J.L., 2003. Sexual factors and Prostate cancer: BLU Int., 92(3): 211-6.
Gupta N.P., Ansari M.S. and Dass S.C., 2005. Transrectal ultrasound guided biopsy for detecting early prostate cancer: an Indian experience: Indian J. Cancer, 42(3): 151-154.

Hoffman R.M., Gilliland F.D., Eley J.W., Harlan L.C., Stephenson R.A., Stanford J.L., Albertson P.C., Hamilton A.S., Hunt W.C. and Potosky A., 2001. Racial and ethnic differences in advanced-stage prostate cancer: The Prostate Cancer Outcomes study: J. Natl. Cancer Inst., 93(5): 388-95.

Hsing A.W. and Comstock G.W., 2009. Serological precursors of cancer: Serum hormones and risk of subsequent prostate cancer: Cancer Epidemiology Biomarkers \& Prevention, 2(1): 27-32.

Jacobs E.J., Rodriquez C., Mondul A.M., Connell C.J., Henly S.J., Calle E.E. and Thun M.J., 2005. A large cohort study of aspirin and other nonsteroidal anti-inflammatory drugs and prostate cancer incidence: J. Natl. Cancer Inst., 97(13): 975-80.

Krishnamoorthy H. and Padmanabha V., 2016. Demography and disease characteristics of prostate cancer in India; Indian Journal of Urology, 32(2): 103-108.

Lija H., Haese A. and Bjork T., 1999. Significance and metabolism of complexed and noncomplexed prostate specific antigen forms, and human glandular kallikrein 2 in clinically localized prostate cancer before and after radical prostatectomy. J. Urol., 162(6): 2029-2031: 1999 Cancer Epidemiology Biomarkers \& Prevention, 9: 1133-1147: Nov 2000.

Lodding P., Aus G. and Bergdahl S., 1998. Characteristics of screening detected prostate cancer in men 50 to 66 years old with 3 to $4 \mathrm{ng} / \mathrm{mL}$. Prostate specific antigen. J. Urol., 159(3): 899-903.

Luboldt H.J., Swoboda A., Börgermann C., Fornara P. and Rübben H., 2001. Early Detection Project Group of the German Society of Urology: Clinical usefulness of free PSA in early detection of prostate cancer. Onkologie., 24(1):33-7.

Malati T. and Rajanikumar G., 2006. Prostate specific antigen in patients of benign prostate hyperptrophy 
and carcinoma Prostate: Indian Journal of Clinical Biochemistry, 21(1):34-40.

Milin T., 1947. Retropubic Urinary Surgery: Carcinima of the Prostate: Radical Retropubic Prostatectomy, Baltimore, Md: Williams and Wilkins, pp.15-7.

Ornstein O.K., Smith D.S., Humprey P.A. and Catalona W.J., 1998. The effect of prostrate volume, age, total prostate specific antigen level and acute inflammation on the percentage of free serum prostate specific antigen level in men without clinically detectable prostate cancer. J. Urol., 159(4): 1234-1237.

Peters U., Leitzmann M.F., Chatterjee N., Wang Y., Albanes D., Gelmann E.P., Friesen M.D., Riboli E. and Hayes R.B., 2007. Serum lycopene, other carotenoids, and prostate cancer risk: a nested case- control study in the prostate, lung, colorectal, and ovarian cancer screening trial: Cancer Epidemiol. Biomarkers Prevention, 16: 962-8.

Platz E.A., Rohrmann S., Pearson J.D., Corrada M.M., Watson D.J., De Marzo A.M., Landis P.K., Metter E.J. and Carter H.B., 2005. Nonsteroidal antiinflamatory drugs and risk of prostate cancer in the Baltimore Longitudinal Study of Aging: Cancer Epidemiol Biomarkers Prev., 14(2): 390-6.
Shannon J., Tewoderos S., Garzotto M., Beer T.M., Derenick R., Palma A. and Farris P.E., 2005. Statins and prostatecancer risk: a case -control study: Am. J. Epidemiol., 162(4): 318-25.

Springhouse, 2005. Professional Guid to Diseases: Staging prostatic cancer: Lippincott Williams and wilkin, vii: 104

Steinberg G.D., Carter B.S., Beaty T.H., Childs B. and Walsh P.C., 1990. Family history and the risk of prostate cancer: Prostate, 17(4): 337-47.

Stephan C., Jung K., Lein M., Sinha P., Schnorr D. and Loening S.A., 1981. Molecular Forms of Prostate specific Antigen and Human Kallikrein 2 as Promising Tools for Early Diagnosis of Prostate Cancer.

Yeole B.B., 2008. Trends in the prostate cancer incidence in India: Asian Pac J. Cancer Prev., 9(1): 141-4.

Zhang W.M., Finne P. and Leinonen J., 1998. Characterization and immunological determination of complex between prostate specific antigen and alpha2-macroglobulin, Clin. Chew, 4(12): 24712479. 